\title{
Mitgliederpresse: Journalismus für die Organisation, PR für die Gesellschaft
}

\author{
Jochen Hoffmann*
}

Der Beitrag entkoppelt PR und Journalismus von spezifischen Handlungsträgern und konzipiert ibr Verbältnis als Meso-Makro-Link. Auf der Basis einer schriftlichen quantitativen Befragung der leitenden Redakteure von national oder sprachregional verbreiteten Mitgliederzeitschriften privater Nonprofit-Organisationen in der Schweiz wird nachgezeichnet, wie sich der Meso-Makro-Link empirisch in sozialen Rollen manifestiert. Deutlich wird, dass sich PR als Organisationsfunktion und Journalismus als Gesellschaftsfunktion nicht wechselseitig ausschließen. Denn als entscheidungsbasierte Sozialsysteme mit hoher Binnenkomplexität zeichnen sich Organisationen durch eine Mehrfachbeteiligung an gesellschaftlichen Funktionssystemen aus. Entsprechend ergeben sich Kompatibilitäten: Eine asymmetrische PR als organisationale Steuerung ist mit einem gesellschaftsorientierten Meinungsjournalismus vereinbar und eine symmetrische $P R$ als organisationales Forum ist mit einem gesellschaftsorientierten Informationsjournalismus kompatibel. Es ist die Funktion der Interdependenzbewältigung, die PR und Journalismus verbindet; die Meso-Makro-Differenz macht sie unterscheidbar.

Schlagwörter: Mitgliederpresse, Organisationskommunikation, Public Relations, Journalismus, Systemtheorie

Die Beziehungen von Public Relations und Journalismus sind ein breit erforschter Untersuchungsgegenstand der Kommunikationswissenschaft (vgl. Weber 1999, Schantel 2000). Mit sog. Determinationsquoten wird gemessen, wie viel Berichterstattung auf PR-Quellen zurückgeht, Selektionsquoten sollen den Erfolg von Pressemitteilungen zeigen. Darüber hinaus werden intervenierende Variablen wie die Konfliktträchtigkeit eines Themas identifiziert (Barth/Donsbach 1992). Trotz zahlreicher Studien bleiben die Befunde aber vielfach kontingent. Das Wissen darüber, unter welchen Bedingungen welche Einflüsse und Abhängigkeiten zwischen PR und Journalismus beobachtet werden können, ist eher fragmentarisch (vgl. Löffelholz 2004).

Eine Ursache für den unbefriedigenden Forschungsstand besteht darin, dass Forschungsdesigns viel konzeptionelle Aufmerksamkeit einer Systematisierung der Beziehungen von PR und Journalismus schenken und eine vorgängige begriffstheoretische Klärung des Unterschieds bzw. der Unterscheidbarkeit von PR und Journalismus vernachlässigen (vgl. Weber 2004: 57-58). Das wird vor allem dann zum Problem, wenn Kommunikationsmedien zum Untersuchungsgegenstand werden, die nicht ,intuitiv“ entweder PR oder Journalismus zugeschlagen werden können. Gemeint ist das Feld der Mitglieder-, Mitarbeiter- und Kundenzeitschriften. Übergreifend lassen sie sich als Stakeholder-Medien (Röttger 2002: 117) bezeichnen, die sich an unterschiedliche Anspruchsgruppen einer Organisation wenden. Ihnen wird vielfach eine professionelle journalistische Aufmachung konstatiert und trotzdem gelten sie zumeist als „PublicRelations-Zeitschriften“" (Bentele 2006).

* Der Autor dankt Olaf Hoffjann (Media Design Hochschule Berlin), Ulrike Röttger (Universität Münster) und Daniela Spranger (Universität Zürich) für kritische und konstruktive Anregungen, die in den Beitrag eingeflossen sind. 
Dieser Beitrag diskutiert gängige Unterscheidungen von PR und Journalismus. Er zeigt damit verbundene Probleme auf, die sich insbesondere für eine Analyse von Stakeholder-Medien ergeben. Es wird ein theoretischer Vorschlag entwickelt, der PR und Journalismus von Handlungsträgern entkoppelt, sodass eine Mitgliederzeitschrift gleichermaßen Ausdruck von PR-Kommunikationen und journalistischen Kommunikationen sein kann. Eine solche Entscheidung erhöht empirische Freiheitsgrade gegenüber theoretischen Vorgaben. Anhand ausgewählter Befunde einer Befragung leitender Redakteure Schweizer Mitgliederzeitschriften ${ }^{1}$ werden beobachtungsleitende Differenzen im Rollenverständnis identifiziert, die es ermöglichen, auf der Grundlage der Meso-Makro-Differenz von Organisation und Gesellschaft sowohl die Unterscheidung wie auch die Kopplung von Public Relations und Journalismus zu beschreiben.

\section{PR als Auftragskommunikation für korporative Akteure}

In der empirischen Forschung ist es üblich, PR und Journalismus anhand ihrer Handlungsträger und Kommunikationsmedien zu unterscheiden. Bentele, Liebert und Seeling (1997) beispielsweise sprechen in ihrem Intereffikationsmodell von PR-Kommunikatoren und journalistischen Kommunikatoren. PR ist demnach das, was ein Pressesprecher sagt und in der Pressemitteilung steht, und Journalismus ist das, was ein Redakteur schreibt und in der Zeitung steht. Der Zeitungsinhalt kann demnach nie selbst PR, sondern nur durch PR-Induktionen beeinflusster Journalismus sein; und der Inhalt einer Pressemitteilung kann nie selbst Journalismus, sondern nur die Simulation von Journalismus mittels gelungener Adaptionen sein.

Diese begriffstheoretische Positionierung schlägt in hohem Maße auf die Operationalisierung von Forschungsfragen durch, die sich der Beziehung von PR und Journalismus widmen. Sie ist aber nur eine pragmatische Prämisse und als solche einer empirischen Prüfung nicht zugänglich. Übernimmt man sie, erübrigt sich die Frage, ob Mitgliederzeitschriften eher PR oder eher Journalismus betreiben. Denn es handelt sich um „Auftragskommunikation“ (Röttger 2002: 118) für spezifische Organisationen, und so werden Stakeholder-Medien a priori zu einem PR-Instrument. Ziel ist die Selbstdarstellung der Organisation (Hardenbicker 1999, Bischl 2000), während in „unabhängigen“ Medien Wirklichkeitsbeschreibung als Fremddarstellung erfolgt.

Ein Verständnis von PR als interessengeleitete Selbstdarstellung zur Imagepflege (Merten/Westerbarkey 1994) lädt dazu ein, PR mit Partikularinteressen und Journalismus an einen Gemeinwohlauftrag zu koppeln (vgl. Baerns 1991). Entsprechend wird PR eher mit einer asymmetrischen persuasiven Kommunikation in Verbindung gebracht, während Journalismus für Meinungsvielfalt und Objektivität stehen soll. In normativer Hinsicht fördert eine solche begriffstheoretische Entscheidung die „Dichotomisierung von moralisch hochwertigem Journalismus und moralisch minderwertiger Öffentlichkeitsarbeit" (Röttger 2000: 110).

Diese Dichotomisierung erscheint fast unvermeidbar angesichts ihrer Kopplung mit einer weiteren beobachtungsleitenden Unterscheidung: PR wird vor allem als Organisationsfunktion konzeptualisiert und Journalismus wird eher als Gesellschaftsfunktion verstanden (ebd., Hoffjann 2001). Es ist aufschlussreich, dass bisherige Versuche, Public Relations auch als Funktionssystem auf der Makroebene zu positionieren (Ronneberger/Rühl 1992, Dernbach 2002), vor allem deshalb gescheitert sind, weil ihre funktionale

1 Vgl. Hoffmann/Spranger 2005; der Autor dankt der IG Mitgliederpresse als Auftraggeber der Studie für die Möglichkeit der wissenschaftlichen Weiterverwertung der Daten. 
Differenz zum Journalismus nicht hinreichend markiert werden konnte (vgl. Röttger 2000: 32-34). Entsprechend besteht wieder die Gefahr einer normativen Überhöhung des Journalismus, weil er im Gegensatz zur PR das gesellschaftliche „Ganze“ im Auge hat. Die folgende Aussage von Ronneberger (1977: 21-22) beinhaltet ein klassisches Deutungsmuster zur Lösung des normativen Dilemmas:

„Wenn also Public Relations-Aktivitäten intentional auf Geltendmachung der eigenen Interessen gerichtet sind und für diese eine möglichst große Resonanz erstreben, so bedeutet dies funktional für das demokratische System, daß die Denkgewohnheiten, sozialen und kulturellen Normen einer Gesellschaft in ihren Teilöffentlichkeiten ständig geprüft, durch Prüfung bestätigt oder gewandelt, jedenfalls ständig in Erinnerung gebracht werden. Dies alles geschieht im durchaus partikulären Interesse.“

Ronneberger transformiert mit dieser Aussage eine pluralistische Deutung von Gesellschaft auf das Verhältnis von PR und Journalismus bzw. Öffentlichkeit. So wie Interessengruppen legitimerweise ihre partikulären Bedürfnisse an den Staat herantragen, die dieser in gesellschaftsrelevante Entscheidungen umsetzt, so speist auch PR seine Kommunikationsangebote in die vom Journalismus verwaltete Öffentlichkeit ein (vgl. Donsbach 1997). Organisationale Partikularinteressen werden normativ aufgewertet, insofern sie in der Summe zum gesellschaftlichen Gemeinwohl beitragen.

Eine pluralistische Deutung von Gesellschaft, die PR legitimiert, benötigt das Konstrukt interessengeleiteter Akteure. Handlungstheoretische Bezüge sind notwendig und sie werden auf eine spezifische Weise mit systemtheoretischem Denken verknüpft, um die Frage zu beantworten, welche durch PR zu legitimierende Interessen Akteure nun genau vertreten. Organisationen werden als korporative Akteure (Willke 2000: 167-188) gefasst, und die „Quelle der Zwecke und Ziele kann dann außerhalb der Organisation verortet werden" (Nassehi 2002: 463). Sie sind in dem gesellschaftlichen Funktionssystem zu finden, welchem sie zuvor untergeordnet worden sind. Ein Unternehmen beispielsweise verfolgt Wirtschaftsinteressen, insofern es dem Funktionssystem Wirtschaft angehört (Martens 2000: 300-305). Entsprechend hat seine PR primär die Aufgabe, zur Legitimation der Gewinnerzielung als Organisationsziel beizutragen (Jarren/Röttger 2004: 27).

Weil nun alle Unternehmen demselben Funktionssystem angehören, gleichen sich auch ihre Organisationsziele, sodass sie zusammengefasst werden können. Systemtheoretisch übersetzt bedeutet das: PR wird zu einer operativen Ausprägung oder sogar zu einem Subsystem des Funktionssystems Wirtschaft und legitimiert dessen Funktion für die Gesellschaft (vgl. Abb. 1). Öffentlichkeitsarbeit wird so auf unterschiedliche Funktionssysteme „verteilt" und muss sich den jeweils geltenden gesellschaftstheoretischen Primärunterscheidungen unterordnen (Marcinkowski 1993: 224-226, Löffelholz 1997: 188). Das heißt: Aus einer zunächst pluralistischen Deutung von Gesellschaft, die korporative Akteure als Handlungsträger von PR benötigt, wird systemtheoretisch ein Meso-Makro-Link konstruiert. Die Folge: Public Relations findet sich fast auf Augenhöhe mit dem Journalismus wieder: Als Leistungssystem der Öffentlichkeit ist Journalismus umgeben von Funktionssystemen, die PR-Operationen ausgebildet haben, um ihre je eigenen Funktionen für die öffentliche Kommunikation zu legitimieren.

Eine Analyse der Mitgliederzeitschriften von Nonprofit-Organisationen hätte auf der Grundlage einer solchen Modellannahme zu klären, welchem Funktionssystem eine herausgebende Organisation zugeschlagen werden kann, damit die Aufgabe der Zeitschrift als PR-Medium identifizierbar wird. Dass es sich um ein PR-Medium handelt, ist dabei theoretisch bereits entschieden. Es wird als Auftragskommunikation und Instru- 


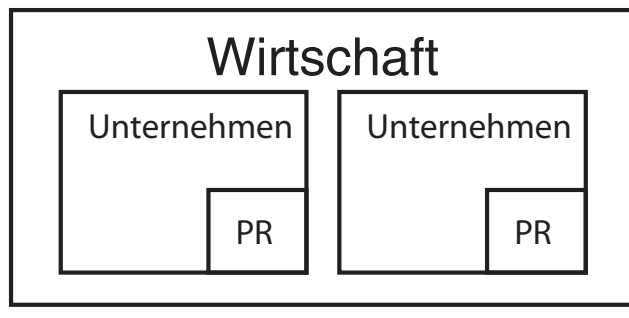

PR als Organisationsfunktion

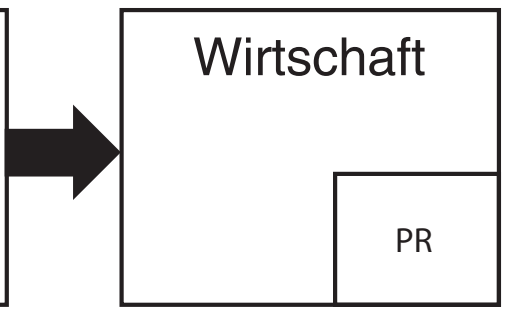

PR als Gesellschaftsfunktion

ment der Zielverwirklichung ebenso exklusiv einem korporativen Akteur zugeordnet, so wie der Akteur exklusiv einem Funktionssystem zugewiesen wird, das wiederum seine Ziele determiniert. Eine solche doppelte Unterordnung lässt empirischen Fragestellungen nicht mehr viel Spielraum. Das nächste Kapitel wird an ausgewählten Beispielen damit verbundene Probleme aufzeigen.

\section{Journalismus und seine Simulation}

Empirische Probleme, die sich aus den dargestellten theoretischen Zuordnungen ergeben, zeigen sich insbesondere bei Inhaltsanalysen von Stakeholder-Medien, und entsprechend gibt es in diesem Feld nur wenige Untersuchungen. In einer Schweizer Studie betrachtet Giovanelli (2003) „Kundenzeitschriften zwischen Public Relations und Journalismus“. Der Untertitel lautet: „Eine Analyse von journalistischen Merkmalen in Schweizer Kundenzeitschriften aus publizistikwissenschaftlicher Perspektive“. Die Reduktion auf journalistische Merkmale bringt das Dilemma der empirischen Forschung zum Ausdruck: Während es gelingt, eine Reihe von Indikatoren für Journalismus etwa aus Darstellungsformen, der Nachrichtenwertforschung oder der Qualitätsdebatte abzuleiten, werden keine davon abgrenzbaren Indikatoren für PR entwickelt. Lediglich eine starke Orientierung auf das eigene Unternehmen gilt als Hinweis auf Öffentlichkeitsarbeit, zugleich wird die Nähe zum Unternehmen als Wirksamkeit des journalistischen Nachrichtenfaktors Relevanz für die Zielgruppe Kunden gedeutet (ebd.: 111-112). Kann demnach derselbe Medieninhalt gleichzeitig Journalismus und PR sein?

Loosen und Meckel (1999) würden diese Frage vermutlich bejahen, insofern sie Greenpeace TV, ein Umweltmagazin, das 1997 auf RTL lief, als „Zwitterform zwischen journalistischem und PR-Angebot“ (ebd.: 389) bezeichnen. Auch in ihrer Inhaltsanalyse gelten Darstellungsformen und Nachrichtenfaktoren als Indikatoren für Journalismus und eine starke Fixierung auf Greenpeace als ein Hinweis auf Public Relations. Festgestellt werden sowohl journalistische Merkmale als auch eine deutliche, wenn auch nach Ansicht der Autorinnen nicht überzogene Selbstdarstellung von Greenpeace.

Auch hier bleibt (positive) Selbstdarstellung der einzige empirische Indikator, der eine inhaltsanalytische Abgrenzung vom Journalismus ermöglicht. „Selbstbeweihräucherung" ohne jede Beachtung journalistischer Regeln wäre in dieser Konsequenz PR in Reinform. Die Relation eines Nullsummenspiels führt aber in eine Sackgasse, gilt doch die Beachtung journalistischer Aufmerksamkeitskriterien geradezu als Merkmal von effektiver Öffentlichkeitsarbeit. Die Abwesenheit von Journalismus in einem Kun- 
denmagazin wäre demnach nicht ein Hinweis auf PR, sondern bestenfalls auf schlechte PR, der die Simulation des Journalismus nicht gelingt. Damit wird von der Empirie etwas eingefordert, was diese nicht leisten kann: die Unterscheidung von Journalismus einerseits und PR als die Simulation von Journalismus andererseits (Hoffjann 2001: 281). Weil dies nicht gelingt, wird der operationalisierbare Weg einer Zuordnung über korporative Akteure als Träger der Kommunikation gegangen: Eine „unabhängige" Zeitschrift betreibt Journalismus, eine Mitgliederzeitschrift simuliert Journalismus.

Aufgrund dieser determinierenden Rolle der Akteure lohnt sich im Weiteren ein Blick auf die Kommunikatorforschung, wobei hier vor allem Studien interessieren, die im direkten Vergleich Selbst- und Fremdwahrnehmungen von PR-Vertretern und Journalisten erheben. Die wenigen, vor allem englischsprachigen Studien zeigen einen hohen Konsens mit Blick auf journalistische Rollenbilder. Sallot, Steinfatt und Salwen (1998) beispielsweise stellen fest, dass „factual accuracy“ sowohl unter Journalisten wie auch PR-Akteuren als wichtigster journalistischer Anspruch gilt. Eine negative Wahrnehmung der PR-Akteure dominiert dagegen unter Journalisten: Der Öffentlichkeitsarbeit gehe es vor allem um positiv getönte (Selbst-)Darstellungen („depicts subject in favorable light“). Erwartungsgemäß widersprechen dem die PR-Akteure: Auch sie nehmen „factual accuracy" als wichtigsten Bezugspunkt ihrer Arbeit wahr. Belz, Talbott und Starck (1989) kommen in einer früheren Studie zu einem ähnlichen Ergebnis und folgern „that journalists and public relations practitioners differ sharply over their perceptions of the public relations role, yet both groups have similar perceptions of the journalistic role." (ebd.: 135) Die negative Fremdwahrnehmung der PR durch Journalisten überrascht wenig, sie ist hilfreich, um sich selbst als "countervailing power" (Sallot/Steinfatt/Salwen 1998: 366) zu positionieren. Überraschend ist aber, dass sich die positiven Selbstbilder so wenig voneinander unterscheiden. So werden beispielsweise auch in PR-Stellenanzeigen insbesondere Interessenten angesprochen, die sich journalistische Qualifikationen zugute halten (Röttger 1997). Das Selbstbild der PR bemüht sich offensichtlich um eine möglichst große Ähnlichkeit mit dem Journalismus.

Wie sind solche Befunde zu interpretieren? Analog zur Ebene der Inhalte könnte die Schlussfolgerung lauten: Die PR-Akteure tun so, als ob sie Journalisten wären, in Wirklichkeit sind sie es aber nicht. Auch eine solche Aussage ist nicht aus empirischen Befunden ableitbar, sondern sie ist die nicht widerlegbare Konsequenz einer akteurstheoretischen Vorabpositionierung. Unabhängig von solchen theoretischen (und normativen) Prämissen bleiben dagegen Hinweise aus Kommunikatorstudien, die darauf hindeuten, dass die Differenz Organisation und Gesellschaft eine auch empirisch relevante beobachtungsleitende Unterscheidung ist. So haben bereits Nayman, McKee und Lattimore (1977: 497) festgestellt: „To a public relations person communication flow within the organization is as important as flow of communications from the organization to the public. This is where our data indicate that professional orientation of the two groups can be distinguished from each other."

In eine ähnliche Richtung weisen Unterschiede zwischen PR-Kodizes und journalistischen Kodizes. In einem Vergleich stellt Raupp (2004: 193) fest, „dass PR-Kodizes hauptsächlich auf die Beziehungen zur auftraggebenden Organisation abgestimmt sind. (...) Journalistische Kodizes dagegen sind von der normativen Annahme des Journalismus als öffentlicher Aufgabe geleitet." Auch das legt nahe, dem Meso-Makro-Link eine zentrale Bedeutung bei der Beantwortung der Frage einer Unterscheidbarkeit von Public Relations und Journalismus zuzuweisen. Das folgende Kapitel wird auf dieser Grundlage einen theoretischen Vorschlag entwickeln, der aber darauf verzichtet, Or- 
ganisationen reifizierend als korporative Akteure Funktionssystemen der Gesellschaft zuzuschlagen.

\section{Der Meso-Makro-Link als System-Umwelt-Beziehung}

Soziale Systeme bestehen nach Luhmann (1997: 80) nicht aus Akteuren oder Handlungen, auch nicht aus Pressemitteilungen oder Zeitungsberichten, sondern aus Kommunikationen. Über die rekursive Verkettung von Kommunikationen entstehen Sinnzusammenhänge als operativ geschlossene Systeme. Wer diesem Paradigma nicht folgt, sondern PR und Journalismus attributiv Handlungsträgern zuordnet, sollte plausibilisieren können, dass die Konstruktion des Akteurs als soziale Entität empirisch nützlich ist und zu einem Erkenntnisgewinn beiträgt. Im Bereich der Mitglieder-, Mitarbeiterund Kundenpresse hat sich gezeigt, dass die theoretische Kopplung von PR an korporative Akteure empirische Forschung gleichermaßen determiniert wie vor unlösbare Probleme stellt. Deshalb ist die Einheit der Organisation zu hinterfragen. Der internen Organisationskommunikation ist mehr Beachtung zu schenken. Insbesondere die soziologische Organisationsforschung zeigt, dass Rationalität, Bürokratie und Hierarchie keine Garanten mehr sind für eine stabile und integrative Organisationsstruktur, die ein einheitliches Auftreten als korporativer Akteur „nach außen“ sicherstellt:

„Mit der Entdeckung dysfunktionaler Programmvorschriften, ineffizienter Regelwerke und mikrosozialer Machtspiele im Gehäuse bürokratischer Organisationen ist der modernen Organisationssoziologie der Grundbegriff strenger Zweckrationalität zunehmend fragwürdig geworden. Hinzu kommt die Beobachtung informaler Strukturen, die die bürokratische, streng formalisierte Ordnungsbildung erweitern, ergänzen und zugleich damit auch unterlaufen. Und schließlich wird mit Blick auf den Einbau dezentraler Aushandlungsprozesse und partizipativer Strukturen auch die Annahme bestritten, dass eine strenge Hierarchisierung der Entscheidungs- und Weisungsketten eine Grundbedingung des Aufbaus komplexer Organisationen darstellt.“ (Kneer 2001: 407)

Aus solchen Beobachtungen kann der theoretische Anspruch abgeleitet werden, „die Selbstbeschreibung des rationalen Akteurs ,Organisation' als einen (durchaus handlungsleitenden!) Mythos zu entlarven.“ (Theis-Berglmair 2005: 42, vgl. auch Berger 1992) Seine Entlarvung macht es notwendig, nicht nur die Makroebene, sondern auch die Mesoebene von handlungstheoretischem Vokabular zu „entrümpeln“ und den korporativen Akteur durch die Organisation als soziales System zu ersetzen: „Die Gleichsetzung der Einheit der Organisation mit der Einheit ihres Zwecks findet mit der Konzeption von Organisationen als soziale Systeme ein Ende." (Theis-Berglmair 2005: 38) Der Mythos des zweckrationalen Akteurs ist somit eine organisationale Selbstbeschreibung, deren Notwendigkeit als Semantik anerkannt bleibt, insofern Organisationssysteme als Kommunikation von Entscheidungen (Luhmann 2000) eine Zurechenbarkeit dieser Entscheidungen benötigen. Sie zeichnen sich durch Adressabilität aus (Fuchs 1997). Einem Unternehmen müssen zweckrationale Entscheidungen zugerechnet werden können; die Wirtschaft als Funktionssystem benötigt eine solche Semantik nicht. Sie kann nicht handeln, muss nicht handeln und muss kein Handeln verantworten (vgl. Kneer 2001: 413-414).

Mit diesem Verweis des korporativen Akteurs in die Welt der Semantik gewinnt nun die interne Kommunikation von Organisationen eine völlig neue Relevanz. Die Organisation ist nicht mehr die soziale Einheit, die Black Box, in die man nicht mehr tiefer hineinsehen kann und auch nicht muss. Ihr Zweck wird auch nicht mehr über die Zuge- 
hörigkeit zu einem Funktionssystem externalisiert, sondern der Zweck selbst wird zum Aushandlungsgegenstand einer komplexen Binnenstruktur (vgl. Serini 1993).

Das wiederum hat erhebliche Konsequenzen für den Meso-Makro-Link: Die Mesoebene ist nicht mehr der Makroebene untergeordnet, die Organisation ist nicht mehr Subsystem eines Funktionssystems, das Unternehmen ist nicht mehr Teil der Wirtschaft, sondern der Meso-Makro-Link wird zu einer System-Umwelt-Relation. Das heißt: Organisationen werden in die Umwelt von Funktionssystemen verlagert (Kneer 2001, Nassehi 2002). Eine solche Vorstellung ist mühsam, weil die in der sozialwissenschaftlichen Theoriebildung beliebten räumlichen Metaphern versagen. Es gibt demnach nicht „eine Art ,Bauprinzip“ sozialer Ordnung, wonach das Gesellschaftssystem sich in Funktionssysteme ausdifferenziert, die sich wiederum aus Organisationssystemen zusammensetzen - ähnlich einem Wohnhaus, das aus unterschiedlichen Etagen besteht, die jeweils wiederum mehrere Zimmer umfassen“ (Kneer 2001: 415-416).

Statt solcher exklusiver Zuordnungen zeichnen sich Organisationen durch „Multireferentialität“ (Bora 2001) aus. Mittels struktureller Kopplungen kommt es zu einer „Mehrfachbeteiligung organisierter Sozialsysteme an gesellschaftlichen Subsystemen“ (Kneer 2001: 412). Ein Universitätsinstitut beispielsweise ist am Wissenschaftssystem, am Erziehungssystem oder auch am Wirtschaftssystem beteiligt. Das Unterschiedliche, „das in der und durch die Organisation subtil aufeinander bezogen wird, ohne zu verschmelzen“ (Nassehi 2002: 455), ergibt sich aus unterschiedlichen Sinnzuweisungen: Die Vorlage eines Forschungsberichts ist für ein Universitätsinstitut eine Entscheidungskommunikation, die Leistungserfüllung dokumentiert und damit weitere Entscheidungskommunikationen erlaubt. Das Institut bleibt indifferent gegenüber dem Wahrheitscode, denn nur das Wissenschaftssystem kann den Forschungsbericht aufgrund dieser Unterscheidung (re)konstruieren. Im Wirtschaftssystem wiederum wird

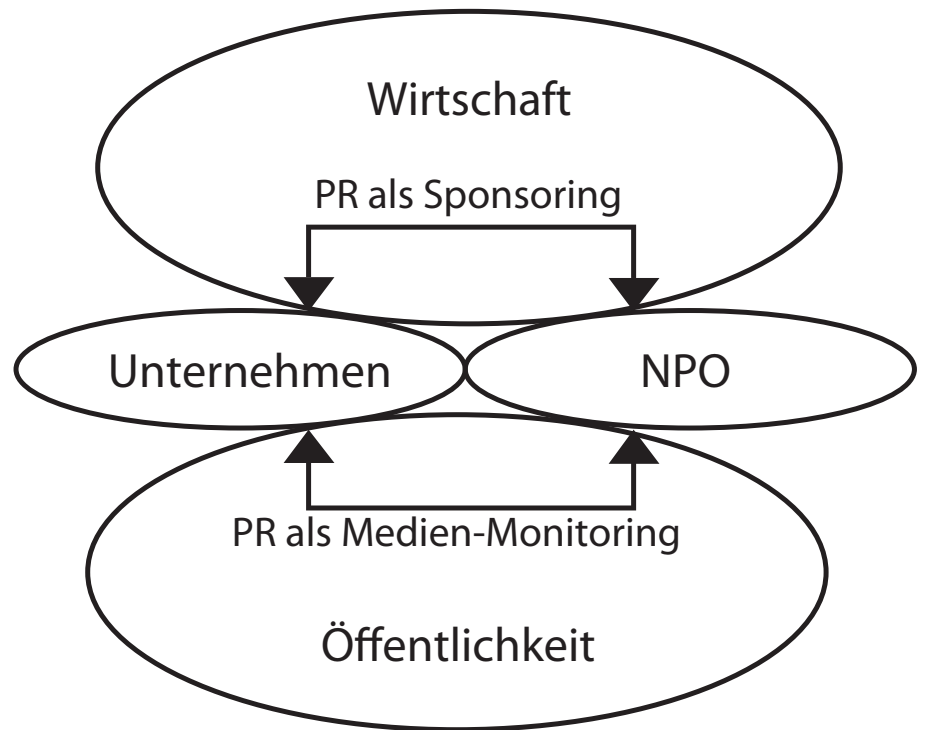


die Leitkodierung Zahlen versus Nicht-Zahlen aktiviert, indem durch den Forschungsbericht Geldleistungen eines Auftraggebers ausgelöst werden, der seinerseits eine Organisation ist.

Letzteres zeigt, dass Funktionssysteme zugleich einen Beitrag zur Interorganisationskommunikation leisten (Kneer 2001: 418-419). Die Abbildung 2 illustriert, wie ein Unternehmen unter „Vermittlung“ des Öffentlichkeitssystems und des Wirtschaftssystems eine Interorganisationsbeziehung eingeht: Das Unternehmen will eine bekannte und angesehene NPO finanziell unterstützen und erhofft sich davon einen Imagetransfer. Über ein Medien-Monitoring, d. h. mit Hilfe des Öffentlichkeitssystems, das Prominenz und Reputation verleiht, werden potenzielle Partner ausgewählt. Wird dann die organisationale Entscheidung eines Sponsoring getroffen, kann sich diese wiederum nicht ohne die Hilfe des Wirtschaftssystems und seiner Leitkodierung Zahlen versus Nicht-Zahlen vollziehen. Dadurch entsteht organisationale wie gesellschaftliche Interdependenz, obwohl die beteiligten Systeme füreinander Umwelt bleiben. Eine direkte Interaktion der Organisationen - wenn auch als Semantik unabdingbar - bleibt unmöglich.

Organisationen versorgen somit die Gesellschaft mit „Zonen dichter Kommunikation " (Nassehi 2004: 110), und die Gesellschaft erleichtert Interorganisationsbeziehungen über operativ geschlossene, aber strukturell gekoppelte Sinnzusammenhänge. Auch dies sprengt wieder die räumliche Metapher: Gesellschaft findet zwar „in“ der Organisation statt, aber als Umwelt der Organisation, nicht als Teil der Organisation. Die Organisation kann demnach nicht mit ihrer Umwelt kommunizieren, sondern die Gesellschaft kehrt gleichsam unerkannt in die Organisation ein. Gesellschaftliche Interdependenzen in Form struktureller Kopplungen werden zu einem Thema der internen Organisationskommunikation, die zugleich Kommunikation der Gesellschaft ist (vgl. Cheney/ Christensen 2001).

Versteht man vor diesem Hintergrund eine Funktion von Organisationen darin, „Ereignisse zum gleichzeitigen Gebrauch in unterschiedlichen Funktionssystemen zu ermöglichen" (Nassehi 2002: 455), wird die Gleichzeitigkeit der Beobachtung von PR und Journalismus in einer Mitgliederzeitschrift möglich, obwohl oder gerade weil beide als unterschiedliche Sinnzusammenhänge modelliert werden. Denn Sinnzusammenhänge sind nicht per definitionem an spezifische Akteure und spezifische Kommunikationsmedien gebunden. Die Mitgliederzeitschrift wird zum Ausdruck einer „internen“ Organisationskommunikation, die über strukturelle Kopplungen auch gesellschaftliche Kommunikation verarbeitet und Interorganisationsbeziehungen bewältigt.

Eine solche Konstruktion des Meso-Makro-Links als System-Umwelt-Beziehung erhöht Freiheitsgrade für eine empirische Forschung, weil Organisationen „Interdependenzmöglichkeiten" (ebd.: 461) nicht nur begrenzen, sondern auch eröffnen. Die Zuordnung von PR zu Akteuren und von Akteuren zu Funktionssystemen muss nicht mehr attributiv und exklusiv vorausgesetzt werden. Wie diese Freiheitsgrade mit dem Ziel eines Erkenntnisgewinns genutzt werden können, soll im Folgenden gezeigt werden. Anhand ausgewählter Befunde einer Befragung von Chefredakteuren Schweizer Mitgliederzeitschriften wird nachgezeichnet, wie sich der Meso-Makro-Link empirisch in sozialen Rollen manifestiert, welche Relationen, Kompatibilitäten und Spannungsfelder entstehen. Begriffstheoretische Schlussfolgerungen mit Blick auf die Unterscheidbarkeit und Gemeinsamkeit von PR und Journalismus werden abschließend gezogen. 


\section{Mitgliederpresse der Schweiz: Sozialisationen und Rollenbilder}

Mitgliederzeitschriften sind periodisch erscheinende Publikationen, die von privaten Nonprofit-Organisationen herausgegeben werden und sich ausschließlich oder teilweise an die Mitglieder der Organisation wenden. Der Bezug der Zeitschrift wird entweder über den Mitgliedsbeitrag entgolten oder sie kann von Mitgliedern abonniert werden. Private Nonprofit-Organisationen bilden den sog. Dritten Sektor, der sich idealtypisch durch eine Äquidistanz von Staat und Markt auszeichnet. Faktisch gibt es aber vielfach eine Abhängigkeit von staatlichen Subventionen und zugleich das legitime Interesse, als Intermediäre staatliches Handeln zu beeinflussen (vgl. Take 2002). Darüber hinaus wird eine mögliche Ökonomisierung des Dritten Sektors diskutiert (vgl. Schauer/Purtschert/ Witt 2002). Die organisationale Herausforderung der „Multireferentialität“ stellt sich somit in besonderem Maße für Nonprofit-Organisationen, sodass die Mitgliederpresse zu einem aufschlussreichen Untersuchungsgegenstand wird.

Den folgenden Befunden liegt eine schriftliche quantitative Befragung der leitenden Redakteure von national oder sprachregional verbreiteten Mitgliederzeitschriften privater Nonprofit-Organisationen in der Schweiz zugrunde. Die Zeitschriften wurden mit dem Ziel größtmöglicher Vollständigkeit sowohl über ein schweizweites Medienverzeichnis („Impressum“) als auch über ein Organisationsverzeichnis („Publicus“) recherchiert, das dem „Oeckl“ in Deutschland vergleichbar ist. Die Befragung wurde von Mai bis Juli 2005 durchgeführt, in die Studie eingegangen sind 373 Fragebögen, die Rücklaufquote beträgt 40 Prozent. $^{2}$

Die Frage, inwieweit sich die Redaktionsleiter eher einer PR-Rolle oder einer journalistischen Rolle zuordnen lassen, kann zunächst auf der Ebene der beruflichen Sozialisationen geprüft werden. Dabei zeigt sich:

- Nur eine Minderheit der Befragten hat ein Studium, eine Ausbildung und/oder eine Weiterbildung im Bereich Kommunikation absolviert (42\%). Von diesen wiederum hat jeder Vierte (24\%) ein allgemeines Angebot in Anspruch genommen (z. B. Publizistik-Studium), während 43 Prozent spezifische Kompetenzen in der PR und 40 Prozent im Journalismus erworben haben. Auf der Ausbildungsebene halten sich PR- und Journalismusbezüge somit die Waage.

- Ein anderes Bild ergibt sich, wenn man danach fragt, welche Ausbildung für den eigenen redaktionellen Nachwuchs empfehlenswert ist. Nur ein Drittel überhaupt empfiehlt die Inanspruchnahme eines Angebots im Bereich Kommunikation (34\%), von diesen hält wiederum ein Drittel eine weitere Spezialisierung nicht für notwendig (33\%). Bezüglich des Verhältnisses von PR und Journalismus gibt es dann aber ein deutliches Ungleichgewicht: Knapp zwei Drittel fordern eine journalistische Ausbildung (65\%), während weniger als jeder Vierte eine Spezialisierung auf PR für sinnvoll hält $(23 \%)$.

- Bei der Berufserfahrung ist das Bild dagegen wieder ausgeglichener: 39 Prozent der Redaktionsleiter geben an, über journalistische Berufserfahrung außerhalb der Mitgliederpresse zu verfügen; berufliche Erfahrungen in der Öffentlichkeitsarbeit können 33 Prozent vorweisen.

- Die Mitgliedschaft in Berufsverbänden schließlich legt wieder eine eindeutige Priorisierung nahe. Von allen Befragten sind 29 Prozent Mitglied in einem Berufsverband, davon 18 Prozent in einer Vertretung für unterschiedliche Kommunikationsberufe.

2 Vgl. zum Feldzugang und methodischen Vorgehen im Detail Hoffmann/Spranger 2005: 2026. 
Nur 5 Prozent sind Mitglied in einem PR-Verband, 76 Prozent dagegen in einer Journalistenvereinigung.

Damit kann festgehalten werden: Während faktische Sozialisationen durch Ausbildung und Berufsweg eine Gleichrangigkeit von PR und Journalismus nahelegen, lassen Indikatoren, die stärker das normative Selbstverständnis berühren - Nachwuchsempfehlung und Berufsverband - eine stärkere Orientierung am Journalismus erkennen. Das deckt sich mit den Befunden aus den aufgeführten Kommunikatorstudien: Eine normativ anspruchsvolle PR sucht die Nähe zu journalistischen Rollenbildern.

Die Vermutung, dass Organisation und Gesellschaft eine nützliche beobachtungsleitende Unterscheidung zur Differenzierung von PR und Journalismus ist, kann vor allem über Zieldefinitionen und Funktionswahrnehmung der Befragten geprüft werden. Zunächst wurden die Redaktionsleiter gebeten, Ziele zu bewerten, die ihnen persönlich mit Blick auf ihre redaktionelle Tätigkeit wichtig oder weniger wichtig sind. Alle vorgegebenen Items, die in die nachfolgende Faktorenanalyse ${ }^{3}$ eingehen, genießen eine hohe Wertschätzung. Auf einer sechsstufigen Skala von eins (= völlig unwichtig) bis sechs (= sehr wichtig) werden Durchschnittswerte zwischen 4,9 („Sachverhalte kritisch aufgreifen und diskutieren“) und 5,5 („einen Beitrag zur Erreichung der Organisationsziele leisten“) erreicht. Trotz dieser geringen Varianz können aber zwei Faktoren unterschieden werden (vgl. Tab. 1). Der eine zeichnet sich durch einen manifesten Organisations-

Tabelle 1: Zieldimensionen redaktioneller Tätigkeit

\begin{tabular}{lcc}
\hline & $\begin{array}{c}\text { Organisations- } \\
\text { bezogene Ziele }\end{array}$ & $\begin{array}{c}\text { Gesellschafts- } \\
\text { bezogene Ziele }\end{array}$ \\
\hline $\begin{array}{l}\text { Gemeinsames Handeln und die lebendige Kommunikati- } \\
\text { on in der Organisation fördern. }\end{array}$ & .79 & .12 \\
$\begin{array}{l}\text { Einen Beitrag zur Erreichung der Organisationsziele } \\
\text { leisten. }\end{array}$ & .74 & -.21 \\
Bedürfnissen der Mitglieder gerecht werden. & .61 & .21 \\
Sachverhalte kritisch aufgreifen und diskutieren. & .14 & .83 \\
Themen und verschiedene Standpunkte möglichst objek- & -.03 & .82 \\
tiv vermitteln. & 31,5 & 29,2 \\
\hline Erklärte Varianz & 1,6 & 1,5 \\
Eigenwert & & \\
\hline
\end{tabular}

Extraktionsmethode: Hauptkomponentenanalyse. Rotationsmethode: Varimax mit Kaiser-Normalisierung. MSA: 0,55. Der MSA-Wert für ein weiteres Item („Meinen Lebensunterhalt sichern“) blieb unter 0,5 und wurde deshalb aus der Faktorenanalyse ausgeschlossen.

3 Die Faktorenanalyse beschreibt Ähnlichkeiten zwischen Variablen. Ausgangsvariablen werden auf möglichst homogene Faktoren reduziert; die Variablen werden gleichsam gebündelt. Damit wird zum einen eine Datenreduktion erreicht, zum anderen werden durch starke und schwache Korrelationen zwischen Variablen die übergreifenden Strukturmerkmale erkennbar. Im Falle der hier gewählten Hauptkomponentenanalyse entspricht der Faktor nicht einer Ursache (formative Faktoren), sondern er ist gleichsam ein Sammelbegriff, der ,hinter' einer größeren Zahl von Einzelvariablen steht, welche untereinander korrelieren (reflektive Faktoren). Die sogenannten Faktorladungen zeigen an, welche Variablen mit welcher Intensität in einen Faktor eingehen. Sie können für jede Variable Werte von -1 bis +1 erreichen. Eine Variable gilt dann als prägend für einen Faktor, wenn sie einen absoluten Wert von mindestens 0,5 erreicht. 
bezug aus, während der andere zwei Orientierungen umfasst, die zumeist als gesellschaftliche Funktionen dem Journalismus zugewiesen werden: objektive Vermittlung von Themen und kritische Diskussion von Sachverhalten.

Die individuellen Zielbestimmungen legen somit organisations- wie gesellschaftsbezogene Rollenbilder nahe, die zwar unterscheidbar sind, aber deshalb nicht zwangsläufig in einen Widerspruch geraten. Der nächste Analyseschritt nimmt eine differenzierte Auswertung beider Ebenen vor. Die Tabelle 2 zeigt zunächst die Gewichtung organisationsbezogener Aufgaben. Von den Redaktoren wird die Bindung der Mitglieder an die Organisation als wichtigste Aufgabe erachtet (5,3). Darüber hinaus sollen die Dienstleistungen der Organisation bekannt gemacht $(5,0)$ sowie deren Standpunkte und Ziele vermittelt werden $(4,9)$. Auch die Mobilisierung der Mitglieder für die Organisationsziele spielt eine wichtige Rolle $(4,5)$. Andere Aufgaben, wie die Erhöhung von Spenden- und Sponsoreneinnahmen aber auch eine Sprachrohrfunktion für die Mitglieder, werden demgegenüber als zweitrangig angesehen.

\section{Tabelle 2: Organisationsbezogene Funktionen}

\begin{tabular}{lc}
\hline Unsere Mitgliederzeitschrift... & Mittelwert \\
\hline ...stärkt die Bindung der Mitglieder an die Organisation. & 5,3 \\
...macht Dienstleistungen der Organisation bekannt. & 5,0 \\
...vermittelt Standpunkte und Ziele der Organisationsleitung. & 4,9 \\
...mobilisiert die Mitglieder für die Organisationsziele. & 4,5 \\
...wirbt neue Mitglieder. & 3,5 \\
...hilft den Organisationsmitgliedern sich zu vernetzen. & 3,3 \\
...vermittelt zwischen unterschiedlichen Interessen innerhalb der Organisation. & 3,1 \\
...ist ein Sprachrohr der Mitglieder gegenüber der Organisationsleitung. & 2,7 \\
...trägt zur Erhöhung der Spendeneinnahmen bei. & 2,5 \\
...trägt zur Erhöhung der Sponsoreneinnahmen bei. & 2,5 \\
\hline
\end{tabular}

$\mathrm{n}=344-367$, Mittelwert auf einer Skala von 1 (= trifft nicht $\mathrm{zu}$ ) bis 6 (= trifft sehr zu).

Um die Frage zu beantworten, ob sich die Items zu übergreifenden Leistungen der Zeitschrift für die Organisation verdichten lassen, wurde wieder eine Faktorenanalyse durchgeführt. Drei Faktoren können identifiziert werden (vgl. Tab. 3). Die vier von den Befragten am wichtigsten erachteten Leistungen prägen gemeinsam den ersten Faktor. Hinter diesen Variablen steht ein Verständnis der Mitgliederzeitschrift als Stenerungsinstrument. In kommunikativer Hinsicht geht es um Top-Down-Prozesse von der Organisation bzw. Organisationsleitung zu den Mitgliedern. Standpunkte, Ziele und Informationen zu Dienstleistungen werden gleichsam von, oben nach unten' vermittelt. Das erhöht die Mitgliederbindung und das Mobilisierungspotenzial.

Der zweite Faktor dagegen wird von Indikatoren geprägt, welche die Mitgliederzeitschrift eher als ein Forum begreifen. Es geht nicht um Top-Down-Prozesse, sondern um horizontale Kommunikationen, die dem Interessenausgleich und der Vernetzung dienen. Hinzu kommt die Sprachrohrfunktion und damit explizit auch Bottom-UpProzesse von den Mitgliedern zur Organisationsleitung. Der dritte Faktor schließlich vereinigt Aspekte von untergeordneter Bedeutung. Die Mitgliederzeitschrift ist hier vor allem Fundraiser - ein Werbemittel, um Spenden, Sponsorengelder und neue Mitglieder $\mathrm{zu}$ akquirieren. Es ist somit vor allem die kommunikative Unterscheidung von Steue- 
Tabelle 3: Dimensionen organisationsbezogener Funktionen

\begin{tabular}{|c|c|c|c|}
\hline Unsere Mitgliederzeitschrift ... & $\begin{array}{l}\text { Steuerungs- } \\
\text { instrument }\end{array}$ & Forum & Fundraiser \\
\hline $\begin{array}{l}\text {... vermittelt Standpunkte und Ziele der Organisations- } \\
\text { leitung. }\end{array}$ & .83 & -.13 & .01 \\
\hline ... macht Dienstleistungen der Organisation bekannt. & .74 & -.02 & .04 \\
\hline ... mobilisiert die Mitglieder für die Organisationsziele. & .69 & -.32 & .20 \\
\hline $\begin{array}{l}\text {... stärkt die Bindung der Mitglieder an die Organisa- } \\
\text { tion. }\end{array}$ & .50 & -.09 & .31 \\
\hline $\begin{array}{l}\text {... hilft den Organisationsmitgliedern sich zu vernet- } \\
\text { zen. }\end{array}$ & .16 & .83 & .07 \\
\hline $\begin{array}{l}\text {... vermittelt zwischen unterschiedlichen Interessen } \\
\text { innerhalb der Organisation. }\end{array}$ & -.00 & .82 & .03 \\
\hline $\begin{array}{l}\ldots \text { ist ein Sprachrohr der Mitglieder gegenüber der } \\
\text { Organisationsleitung. }\end{array}$ & .32 & .75 & .19 \\
\hline ... trägt zur Erhöhung der Spendeneinnahmen bei. & .15 & -.12 & .80 \\
\hline ... trägt zur Erhöhung der Sponsoreneinnahmen bei. & .08 & -.09 & .79 \\
\hline ... wirbt neue Mitglieder. & .09 & -.25 & .68 \\
\hline Erklärte Varianz & 21,3 & 21,2 & 18,8 \\
\hline Eigenwert & 2,1 & 2,1 & 1,9 \\
\hline
\end{tabular}

Extraktionsmethode: Hauptkomponentenanalyse. Rotationsmethode: Varimax mit Kaiser-Normalisierung. MSA: 0,77

rungs- und Forumsfunktion, der empirisch ein beobachtungsleitendes Potenzial zugewiesen werden kann. Ergänzt wird sie um das nachrangige Fundraising.

Die individuellen Zielbestimmungen der Befragten haben gezeigt, dass sich Organisations- und Gesellschaftsorientierung nicht ausschließen müssen. Welche Relevanz wird also gesellschaftsbezogenen Funktionen zugewiesen, wie sie üblicherweise in Journalistenbefragungen erhoben werden, ${ }^{4}$ um Rollenbilder zu identifizieren? Das Ergebnis zeigt, dass in einer objektiven, sorgfältigen, präzisen Vermittlung komplexer Sachverhalte die wichtigste Aufgabe einer Mitgliederzeitschrift gesehen wird (vgl. Tab. 4). Am unwichtigsten ist dagegen der Einsatz für Schwache und Benachteiligte sowie die Zeitschrift als Orientierung vor Abstimmungen und Wahlen. Die anderen Items bilden ein breites Mittelfeld mit Durchschnittswerten, die von 3,7 bis 4,6 vergleichsweise eng beieinander liegen.

Auch diese Funktionswahrnehmungen können mittels Faktorenanalyse zu Rollenbildern verdichtet werden (vgl. Tab. 5). Zum einen zeigen sich wieder die beiden Selbstverständnisse, deren Relevanz in der deutschsprachigen Journalismus-Forschung zu teils heftigen Diskussionen geführt haben: (objektiver) Informationsjournalismus contra (kritischer) Meinungsjournalismus (vgl. Löffelholz 2003: 47-49). Der dritte Typus ist ein Rollenbild, welches erst seit wenigen Jahren verstärkt diskutiert wird. Hier steht die

4 Vgl. für die Schweiz Marr u. a. 2001, für Deutschland Weischenberg/Malik/Scholl 2006 sowie Weaver u. a. 2007 als die zum dritten Mal durchgeführte, international wichtigste Referenzstudie aus den USA. 
Tabelle 4: Gesellschaftsbezogene Funktionen

\begin{tabular}{lc}
\hline Unsere Mitgliederzeitschrift... & Mittelwert \\
\hline ...vermittelt Themen möglichst objektiv. & 5,1 \\
...analysiert komplexe Sachverhalte sorgfältig und präzise. & 4,8 \\
...geht auf Bedürfnisse der Leserinnen und Leser ein. & 4,6 \\
...trägt zur Meinungsbildung bei. & 4,3 \\
...zeigt Missstände auf. & 4,0 \\
...bringt wichtige Themen in die öffentliche Diskussion. & 3,9 \\
...ist unterhaltsam für die Leserinnen und Leser. & 3,9 \\
...fungiert als Ratgeberin für Leserinnen und Leser. & 3,8 \\
...zeigt auf, wo man sich engagieren kann. & 3,7 \\
...bietet eine Plattform für unterschiedliche Akteure. & 3,7 \\
...setzt sich für Schwache und Benachteiligte ein. & 3,1 \\
...bietet eine wichtige Orientierung vor Abstimmungen und Wahlen. & 2,4 \\
\hline
\end{tabular}

$\mathrm{n}=347-367$, Mittelwert auf einer Skala von 1 (= trifft nicht $\mathrm{zu}$ ) bis 6 (= trifft sehr zu).

strikte Orientierung am Leser im Zentrum. Seine Bedürfnisse gelten als entscheidend. Ziel ist es, ihm Lebenshilfe und Unterhaltung zu bieten. Das kann zunächst als Hinweis für eine Aufwertung von Bottom-Up-Kommunikationen gesehen werden, weil der Rezipient ins Zentrum rückt. Das Rollenbild ist jedoch auch kompatibel mit dem Leser als einem passiven Konsumenten, der nach ökonomischen Kriterien mit den Angeboten eines „Marketingjournalismus“ (Weischenberg 1995: 334) bedient wird. Insofern kann die Leserorientierung nicht parallelisiert werden mit Bottom-Up-Kommunikationen, wie sie im Kontext der organisationalen Forumsfunktion identifiziert wurden. Denn auch eine Kompatibilität mit der Fundraising-Funktion ist denkbar. Aus diesem Grund wird die „klassische“ Gegenüberstellung von Informations- und Meinungsorientierung als empirisch relevante beobachtungsleitende Unterscheidung der Redakteure angenommen.

Der nächste Schritt prüft Einflüsse der berufsbiographischen Prägungen der Befragten auf Zieldefinitionen und die organisations- wie gesellschaftsbezogenen Funktionswahrnehmungen. Befunde können Hinweise auf plausible Zuordnungen von PR und Journalismus zu den Rollenbildern ergeben. Es zeigen sich aber nur wenige bivariate Zusammenhänge:

- Aus- und Weiterbildung: Wer eine Aus- und Weiterbildung in der PR, nicht aber im Journalismus wahrgenommen hat, betont stärker organisationsbezogene Ziele des redaktionellen Handelns. $(\mathrm{p}=.025)$

- Nachrouchsempfehlung: Wer dem Nachwuchs eine PR-Ausbildung, nicht aber eine journalistische Ausbildung empfiehlt, betont stärker die organisationsbezogene Funktion des Fundraisers. $(\mathrm{p}=.015)$

- Berufserfahrung: Eine unterschiedliche Berufserfahrung in PR und Journalismus hat keinen signifikanten Einfluss auf Zieldefinitionen und Funktionswahrnehmung.

- Berufsverband: Wer in einem journalistischen Berufsverband Mitglied ist, betont stärker die organisationsbezogene Forumsfunktion und die gesellschaftsbezogene Informationsorientierung. ( $\mathrm{p}=.003 \mathrm{bzw} . \mathrm{p}=.019$, jew. Mann-Whitney-U-Test) 
Tabelle 5: Dimensionen gesellschaftsbezogener Funktionen

\begin{tabular}{|c|c|c|c|}
\hline Unsere Mitgliederzeitschrift... & $\begin{array}{l}\text { Meinungs- } \\
\text { orientierung }\end{array}$ & $\begin{array}{l}\text { Leser- } \\
\text { orientierung }\end{array}$ & $\begin{array}{l}\text { Informati- } \\
\text { onsorientie- } \\
\text { rung }\end{array}$ \\
\hline $\begin{array}{l}\text {... bringt wichtige Themen in die öffentliche } \\
\text { Diskussion. }\end{array}$ & .80 & .00 & .20 \\
\hline ... zeigt Missstände auf. & .76 & -.03 & .02 \\
\hline ... trägt zur Meinungsbildung bei. & .74 & .11 & .22 \\
\hline $\begin{array}{l}\text {... bietet eine wichtige Orientierung vor Abstim- } \\
\text { mungen und Wahlen. }\end{array}$ & .71 & -.04 & .05 \\
\hline ... zeigt auf, wo man sich engagieren kann. & .65 & .25 & -.33 \\
\hline ... setzt sich für Schwache und Benachteiligte ein. & .53 & .26 & -.48 \\
\hline $\begin{array}{l}\text {... geht auf Bedürfnisse der Leserinnen und Leser } \\
\text { ein. }\end{array}$ & -.04 & .74 & .20 \\
\hline $\begin{array}{l}\text {... fungiert als Ratgeberin für Leserinnen und } \\
\text { Leser. }\end{array}$ & .13 & .66 & -.01 \\
\hline ... ist unterhaltsam für die Leserinnen und Leser. & -.06 & .64 & -.08 \\
\hline $\begin{array}{l}\text {... bietet eine Plattform für unterschiedliche } \\
\text { Akteure. }\end{array}$ & .19 & .47 & .28 \\
\hline $\begin{array}{l}\text {... analysiert komplexe Sachverhalte sorgfältig } \\
\text { und präzise. }\end{array}$ & .24 & .03 & .72 \\
\hline ... vermittelt Themen möglichst objektiv. & -.02 & .22 & .69 \\
\hline Erklärte Varianz & 25,8 & 15,1 & 12,9 \\
\hline Eigenwert & 3,1 & 1,8 & 1,6 \\
\hline
\end{tabular}

Extraktionsmethode: Hauptkomponentenanalyse. Rotationsmethode: Varimax mit Kaiser-Normalisierung. MSA: 0,77

Lediglich der Einfluss des Indikators Aus- und Weiterbildung spricht dafür, dass PRAkteure eine primär organisationsbezogene und Journalisten eine primär gesellschaftsbezogene Perspektive auf ihr berufliches Handeln einnehmen. Beim Indikator Nachwuchsempfehlung kann dagegen nur bei einer der drei Organisationsfunktionen ein Einfluss der PR-Orientierung festgestellt werden und die Berufserfahrung bleibt gänzlich irrelevant. Interessant ist schließlich, dass sich die Mitgliedschaft in einem journalistischen Berufsverband positiv sowohl auf eine gesellschaftsbezogene wie auch auf eine organisationsbezogene Funktion auswirkt.

Es finden sich demnach nur wenige Hinweise dafür, dass eine PR-Sozialisation per se die Organisationsorientierung und eine journalistische Sozialisation die Gesellschaftsorientierung des redaktionellen Handelns fördert. Die Parallelisierung von Organisation und Gesellschaft mit $P R$ und Journalismus erzwingt keine sich ausschließende Alternative. Vielmehr muss von spezifischen Kompatibilitäten und Inkompatibilitäten ausgegangen werden. Um welche es sich dabei handelt, wird deutlich, wenn die Zieldefinitionen der Befragten mit ihren Funktionswahrnehmungen korreliert werden (vgl. Abb. 3).

Erwartungsgemäß besteht ein signifikant positiver Zusammenhang zwischen einer organisationsbezogenen Zieldefinition und allen drei organisationsbezogenen Funktionswahrnehmungen. Umgekehrt korreliert die gesellschaftsbezogene Zieldefinition mit 

Abbildung 3: Zusammenhang zwischen organisations- und gesellschaftsbezogenen
Rollenbildern

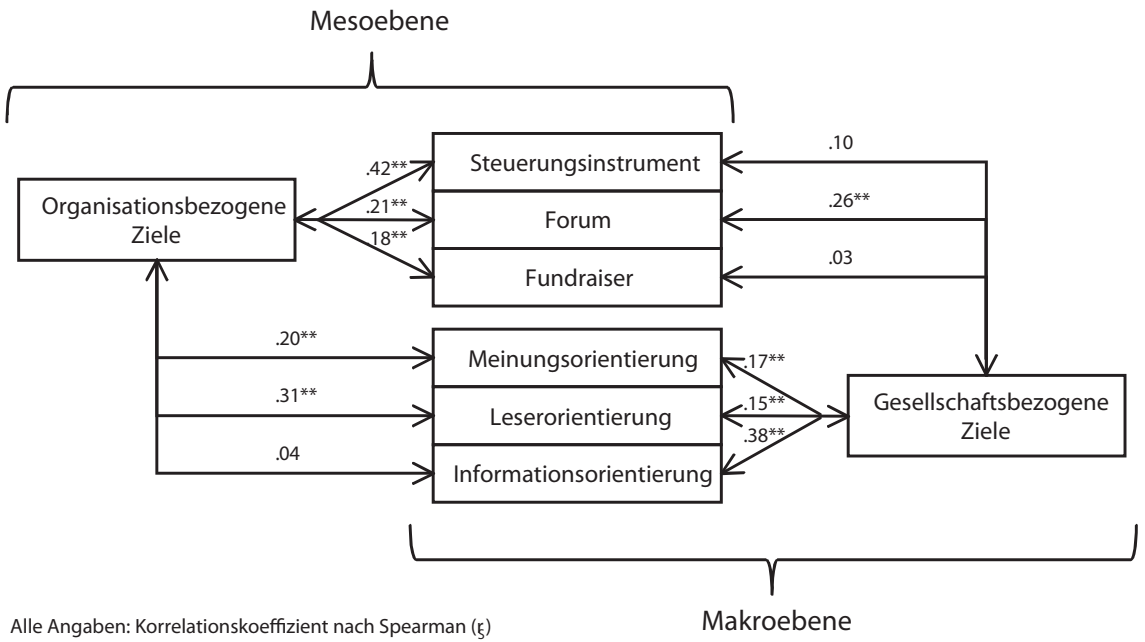

den journalistisch ausgerichteten, gesellschaftsbezogenen Funktionswahrnehmungen. Das eigentlich Interessante ist die Tendenz zu einer Polarisierung zwischen zwei Rollenbildern:

- Die Zeitschrift als Steuerungsinstrument korreliert besonders stark mit der Organisationsorientierung des Befragten $\left(\mathrm{r}_{\mathrm{S}}=.42 \%\right.$ ) und nicht mit einer Gesellschaftsorientierung.

- Informationsorientierung korreliert besonders stark mit der Gesellschaftsorientierung des Befragten $\left(\mathrm{r}_{S}=.38^{* *}\right)$ und nicht mit einer Organisationsorientierung.

PR als Steuerungsinstrument für die Organisation einerseits versus Informationsjournalismus für die Gesellschaft andererseits wären somit Pole, die am ehesten Inkompatibilitäten erzeugen. Zugleich lassen sich aber auch Kompatibilitäten zwischen Meso- und Makroebene ausmachen:

- Meinungs- und Leserorientierung korrelieren positiv mit der Organisationsorientierung $\left(\mathrm{r}_{\mathrm{S}}=.20 * \%\right.$ bzw. $\left..31 \% *\right)$.

- Die Forumsfunktion korreliert positiv mit der Gesellschaftsorientierung $\left(\mathrm{r}_{\mathrm{S}}=\right.$ $.26 * *)$.

Damit sprechen die Befunde insgesamt nicht dafür, dass PR und Journalismus völlig unterschiedliche Welten sind, die gleichsam in der Mitgliederpresse unversöhnlich aufeinanderprallen und zugunsten einer Seite und einem vorab definierten Organisationsinteresse entschieden werden müssen. Ein gewisses Spannungsverhältnis lässt sich lediglich zwischen organisationalen Steuerungsansprüchen und gesellschaftlichen Informationsansprüchen ausmachen. Das bedeutet jedoch nicht, dass damit zugleich auch die geeignete begriffstheoretische Antwort zur Unterscheidbarkeit von PR und Journalismus gegeben ist. Kompatibilitäten zwischen Meso- und Makroebene und die doch überraschend geringen Einflüsse berufsspezifischer Sozialisationen lassen ein vielschichtigeres Verhältnis erwarten, das im folgenden Kapitel abschließend skizziert wird. 


\section{Schlussfolgerungen}

Mitgliederzeitschriften, so eine gängige Deutung, gehören zu PR-Medien, die der Selbstdarstellung einer Organisation und der Legitimation des Organisationsziels gegenüber spezifischen Anspruchsgruppen dienen. Das Organisationsziel wiederum leitet sich aus der Zugehörigkeit zu einem übergeordneten Funktionssystem ab. Daraus ergibt sich die Unterscheidung von PR und Journalismus, insofern letzterer als eigenständiges gesellschaftliches Leistungssystem der Herstellung aktueller Wirklichkeiten dient (Scholl/ Weischenberg 1998). Die pluralistische Kopplung der PR an eine Organisation als einem korporativen Akteur führt bei der Erforschung von Stakeholder-Medien jedoch zu Problemen, weil Journalismus von seiner Simulation empirisch nicht zu unterscheiden ist. Aus diesem Grund wird die Entkopplung von PR und Journalismus von möglichen Handlungsträgern vorgeschlagen. Versteht man Organisationen als entscheidungsbasierte Sozialsysteme mit Mehrfachbeteiligung an gesellschaftlichen Funktionssystemen, entsteht eine komplexe Binnenstruktur, die auf der Ebene der Mitgliederpresse die Gleichzeitigkeit von PR und Journalismus als empirische Möglichkeit vorsieht.

Empirische Befunde aus einer Befragung der leitenden Redakteure Schweizer Mitgliederzeitschriften unterstützen diese theoretische Herangehensweise. Zunächst können eher organisations- und eher gesellschaftsbezogene Ziele redaktionellen Handelns identifiziert werden. Beide Orientierungspunkte schließen sich nicht prinzipiell aus. Inkompatibilitäten ergeben sich vielmehr aus einer zweiten Unterscheidung, die gleichsam "quer" zur Meso-Makro-Differenz liegt. Es ist das Spannungsfeld von symmetrischer und asymmetrischer Kommunikation. Auf der Organisationsebene macht es einen Unterschied, ob man die Mitgliederzeitschrift vor allem als ein Steuerungsinstrument oder als ein Forum der Mitgliedschaft versteht. Auf der Gesellschaftsebene macht es einen Unterschied, ob man einen persuasiven Meinungsjournalismus betreibt oder sich primär um einen objektiven Informationsjournalismus bemüht. Objektivität lässt sich journalistisch als Perspektivenvielfalt herstellen (ebd.: 181), die prozessual mittels symmetrischer Kommunikationen gefördert werden kann.

Damit können zwei empirisch relevante beobachtungsleitende Unterscheidungen ausgemacht werden: Organisations- und Gesellschaftsbezug sowie asymmetrische und symmetrische Kommunikation. Welche der beiden Unterscheidungen ist besser geeignet, um zwischen PR und Journalismus zu differenzieren? Die Tabelle 6 schlägt eine Zuordnung vor, die gemäß der theoretischen Ausführungen die Meso-Makro-Unterscheidung als entscheidend positioniert. Die soziale Inkompatibilität von asymmetrischer und symmetrischer Kommunikation muss demnach innerbalb der PR und innerbalb des Journalismus als Inklusionsproblem bewältigt werden.

\section{Tabelle 6: Beobachtungsleitende Unterscheidungen für $P R$ und Journalismus}

\begin{tabular}{lll}
\hline & Organisationsbezug & Gesellschaftsbezug \\
\hline Asymmetrisch & PR als Steuerungsinstrument & Meinungsjournalismus \\
Symmetrisch & PR als Forum & Informationsjournalismus \\
\hline
\end{tabular}

Würde man stattdessen asymmetrische und symmetrische Kommunikation mit der Unterscheidung von PR und Journalismus parallelisieren, wäre die organisationale Forumsfunktion keine Leistung von PR, sondern ein Ausdruck von Journalismus. Und Meinungsjournalismus wäre kein Journalismus, sondern Public Relations. Es entstünde 
wieder das Problem, dass mit der Kopplung von Public Relations an asymmetrische Kommunikationen bereits auf der Begriffsebene die Möglichkeit einer „symmetrischen PR“ im Sinne von Grunig und Hunt (1984) oder einer „verständigungsorientierten Öffentlichkeitsarbeit" von Burkart (1993) ausgeschlossen wird. Die empirisch festgestellte normative Orientierung der PR an journalistischen Rollenbildern könnte damit erklärt werden; sie würde aber zugleich als unlösbares professionelles Identitätsproblem zementiert. PR würde wieder a priori moralisch ab- und Journalismus würde aufgewertet.

Weil normative Zuschreibungen höchstens Folge, nicht aber begriffstheoretische Voraussetzung empirischer Erkenntnis sein können, erscheint es vielversprechender, an Konzepte von PR als Organisationsfunktion anzuknüpfen. Es wird jedoch vermieden, Organisationen als korporative Akteure zu fassen. Stattdessen wird die Gesellschaft in der Organisation als Ausdruck struktureller Kopplungen zugelassen. Empirisch bedeutet das, dass die Kompatibilität zwischen einer organisationalen Forumsfunktion der PR und einem Informationsjournalismus genauso zu beachten ist wie der gemeinsame persuasive Charakter von PR als Steuerungsfunktion und einem Meinungsjournalismus. Stakeholder-Ansätze, welche die Differenz von interner und externer Kommunikation in Frage stellen (Karmasin 2005), oder ein Verständnis von PR als Interdependenzmanagement (zuletzt Hoffmann/Röttger/Jarren 2007) könnten einen hierfür geeigneten übergreifenden theoretischen Rahmen abgeben. Sie sind aber voraussetzungsvoll. Interdependenzmanagement als PR-Leistung setzt die „Handhabung der Unterscheidung von Selbstreferenz und Fremdreferenz" (Nassehi 2002: 466) voraus und damit eine „Selbstbeobachtung, die nicht den eigenen Selbstbeschreibungen zum Zwecke der Präsentationspolitik (Werbung, Broschüren) oder der Motivierung von Personal (Leitbilder, corporate identities) auf den Leim geht" (ebd.: 467).

Einzufordern von Public Relations wäre somit ein reflexiver Umgang mit selbst erzeugten Organisationsbeschreibungen. Die damit erzeugte Kontingenz kann bewältigt werden, wenn der systemische Bezugspunkt unhinterfragt bleibt. Gesellschaftliche Interdependenzen sind für PR nur dann relevant, wenn sie die Entscheidungskommunikation der Organisation berühren. Darin liegt der entscheidende Unterschied zum Journalismus als Gesellschaftsfunktion. Entscheidungsrelevanz als organisationales Selektionskriterium eröffnet eine völlig andere Perspektive der Umweltbeobachtung als der Aktualitätscode des Journalismus, und zugleich weist Interdependenzmanagement als organisationales Programm einmal mehr erstaunliche Parallelen zum Journalismus als Gesellschaftsfunktion auf. Dort spricht man von einer notwendigen Synchronisation der Funktionssysteme (Görke 1999), vom journalistischen „Kommunizieren über die Mehrsystemzugehörigkeit von Ereignissen“ (Kohring 2004: 197). Die Funktion der Interdependenzbewältigung kann demnach als die entscheidende Gemeinsamkeit von Public Relations und Journalismus gesehen werden. Nur die Warte bleibt eine andere: Der Journalismus leistet für die Gesellschaft, was die PR für die Organisation leistet. Journalismus ist PR für die Gesellschaft, PR ist Journalismus für die Organisation. Die vornehmste Aufgabe von Public Relations und von Journalismus könnte demnach darin bestehen, „Mehrdeutigkeit in mehrdeutiger Weise anzupacken“ (Weick 1998: 270).

\section{Literatur}

Baerns, Barbara (1991): Öffentlichkeitsarbeit oder Journalismus? Zum Einfluß im Mediensystem, 2. Aufl., Köln.

Barth, Henrike / Donsbach, Wolfgang (1992): Aktivität und Passivität von Journalisten gegenüber 
Public Relations. Eine Fallstudie am Beispiel von Pressekonferenzen zu Umweltthemen. In: Publizistik 37, 151-165.

Belz, Andrew / Talbott, Albert T. / Stark, Kenneth (1989): Using Role Theory to Study Cross Perceptions of Journalists and Public Relations Practitioners. In: Grunig, James E. / Grunig, Larissa A. (Hg.): Public Relations Research Annual, 1, Hillsdale, 125-139.

Bentele, Günter (2006): Public-Relations-Zeitschriften. In: Bentele, Günter / Brosius, Hans-Bernd / Jarren, Otfried (Hg.): Lexikon Kommunikations- und Medienwissenschaft, Wiesbaden, 232.

Bentele, Günter / Liebert, Thomas / Seeling, Stefan (1997): Von der Determination zur Intereffikation. Ein integriertes Modell zum Verhältnis von Public Relations und Journalismus. In: Bentele, Günter / Haller, Michael (Hg.): Aktuelle Entstehung von Öffentlichkeit. Akteure - Strukturen - Veränderungen, Konstanz, 225-250.

Berger, Ulrike (1992): Rationalität, Macht und Mythen. In: Küpper, Willi / Ortmann, Günther (Hg.): Mikropolitik. Rationalität, Macht und Spiele in Organisationen, 2. Aufl., Opladen, 115130.

Bischl, Katrin (2000): Die Mitarbeiterzeitung. Kommunikative Strategien der positiven Selbstdarstellung von Unternehmen, Wiesbaden.

Bora, Alfons (2001): Öffentliche Verwaltung zwischen Recht und Politik. Die Multireferentialität organisatorischer Kommunikation. In: Tacke, Veronika (Hg.): Organisation und gesellschaftliche Differenzierung, Opladen, 170-191.

Burkart, Roland (1993): Public Relations als Konfliktmanagement. Ein Konzept für verständigungsorientierte Öffentlichkeitsarbeit. Untersucht am Beispiel der Planung von Sonderabfalldeponien in Niederösterreich, Wien.

Cheney, George / Christensen, Lars Thoger (2001): Organizational Identity. Linkages between Internal and External Communication. In: Jablin, Fredric M. / Putnam, Linda L. (Hg.): The New Handbook of Organizational Communication, London, 231-269.

Dernbach, Beatrice (2002): Public Relations als Funktionssystem. In: Scholl, Armin (Hg.): Systemtheorie und Konstruktivismus in der Kommunikationswissenschaft, Konstanz, 129-145.

Donsbach, Wolfgang (1997): Legitimität und Effizienz von Public Relations. In: Donsbach, Wolfgang (Hg.): Public Relations in Theorie und Praxis: Grundlage und Arbeitsweise der Öffentlichkeitsarbeit in verschiedenen Funktionen, München, 7-20.

Fuchs, Peter (1997): Adressabilität als Grundbegriff der soziologischen Systemtheorie. In: Soziale Systeme 3, 55-79.

Giovanelli, Iris (2003): Kundenzeitschriften zwischen Public Relations und Journalismus. Eine Analyse von journalistischen Merkmalen in Schweizer Kundenzeitschriften aus publizistikwissenschaftlicher Perspektive, Unveröffentl. Lizenziatsarbeit, Zürich.

Görke, Alexander (1999): Risikojournalismus und Risikogesellschaft. Sondierung und Theorieentwurf, Wiesbaden.

Grunig, James E. / Hunt, Todd (1984): Managing Public Relations, New York.

Hardenbicker, Markus (1999): Kundenzeitschriften. Ein linguistischer Beschreibungsansatz auf kommunikationsanalytischer Grundlage, Frankfurt/Oder.

Hoffjann, Olaf (2001): Journalismus und Public Relations. Ein Theorieentwurf der Intersystembeziehungen in sozialen Konflikten, Wiesbaden.

Hoffmann, Jochen / Röttger, Ulrike / Jarren, Otfried (2007): Structural Segregation and Openness: Balanced Professionalism for Public Relations. In: Studies in Communication Sciences 7 (1), 125-146.

Hoffmann, Jochen / Spranger, Daniela (2005): Die Mitgliederpresse von Nonprofit-Organisationen in der Schweiz, Unveröffentl. Forschungsbericht, Bern.

Jarren, Otfried / Röttger, Ulrike (2004): Steuerung, Reflexierung und Interpenetration: Kernelemente einer strukturationstheoretisch begründeten PR-Theorie. In: Röttger, Ulrike (Hg.): Theorien der Public Relations. Grundlagen und Perspektiven der PR-Forschung, Wiesbaden, 25-45.

Karmasin, Matthias (2005): Stakeholder Management als Ansatz der PR. In: Bentele, Günter / Fröhlich, Romy / Szyszka, Peter (Hg.): Handbuch der Public Relations. Wissenschaftliche Grundlagen und berufliches Handeln. Mit Lexikon, Wiesbaden, 268-296. 
Kneer, Georg (2001): Organisation und Gesellschaft. Zum ungeklärten Verhältnis von Organisations- und Funktionssystemen in Luhmanns Theorie sozialer Systeme. In: Zeitschrift für Soziologie 30, 407-428.

Kohring, Matthias (2004): Journalismus als soziales System. Grundlagen einer systemtheoretischen Journalismustheorie. In: Löffelholz, Martin (Hg.): Theorien des Journalismus. Ein diskursives Handbuch, 2. Aufl., Wiesbaden, 185-200.

Löffelholz, Martin (1997): Dimensionen struktureller Kopplung von Öffentlichkeitsarbeit und Journalismus. Überlegungen zur Theorie selbstreferentieller Systeme und Ergebnisse einer repräsentativen Studie. In: Bentele, Günter / Haller, Michael (Hg.): Aktuelle Entstehung von Öffentlichkeit. Akteure - Strukturen - Veränderungen, Konstanz, 187-208.

Löffelholz, Martin (2003): Kommunikatorforschung: Journalistik. In: Bentele, Günter / Brosius, Hans-Bernd / Jarren, Otfried (Hg.): Öffentliche Kommunikation. Handbuch Kommunikations- und Medienwissenschaft, Wiesbaden, 28-53.

Löffelholz, Martin (2004): Ein privilegiertes Verhältnis. Theorien zur Analyse der Inter-Relationen von Journalismus und Öffentlichkeitsarbeit. In: ders. (Hg.): Theorien des Journalismus. Ein diskursives Handbuch, 2. Aufl., Wiesbaden, 471-485.

Loosen, Wiebke / Meckel, Miriam (1999): Journalismus in eigener Sache. Veränderungen im Verhältnis von Journalismus und Public Relations am Beispiel von Greenpeace TV. In: Rundfunk und Fernsehen 47, 379-392.

Luhmann, Niklas (1997): Die Gesellschaft der Gesellschaft, 2 Bde, Frankfurt a. M.

Luhmann, Niklas (2000): Organisation und Entscheidung, Wiesbaden.

Marcinkowski, Frank (1993): Publizistik als autopoietisches System. Politik und Massenmedien. Eine systemtheoretische Analyse, Opladen.

Marr, Mirko u. a. (2001): Journalisten in der Schweiz. Eigenschaften, Einstellungen, Einflüsse, Konstanz.

Martens, Wil (2000): Organisation und gesellschaftliche Teilsysteme. In: Ortmann, Günter / Sydow, Jörg / Türk, Klaus (Hg.): Theorien der Organisation. Die Rückkehr der Gesellschaft, 2. Aufl., Wiesbaden, 263-311.

Merten, Klaus / Westerbarkey, Joachim (1994): Public Opinion und Public Relations. In: Merten, Klaus / Schmidt, Siegfried J. / Weischenberg, Siegfried (Hg.): Die Wirklichkeit der Medien. Eine Einführung in die Kommunikationswissenschaft, Opladen.

Nassehi, Armin (2002): Die Organisationen der Gesellschaft. Skizze einer Organisationssoziologie in gesellschaftstheoretischer Absicht. In: Allmendinger, Jutta / Hinz, Thomas (Hg.): Organisationssoziologie, Wien, 443-478.

Nassehi, Armin (2004): Die Theorie funktionaler Differenzierung im Horizont ihrer Kritik. In: Zeitschrift für Soziologie 33, 98-118.

Nayman, Oguz / McKee, Blaine K. / Lattimore, Dan L. (1977): PR Personnel and Print Journalists: A Comparison of Professionalism. In: Journalism Quarterly 54, 492-497.

Raupp, Juliana (2004): Berufsethische Kodizes als Konfliktvermeidungsprogramme. PR-Kodizes und Pressekodizes im Vergleich. In: Altmeppen, Klaus-Dieter / Bentele, Günter / Röttger, Ulrike (Hg.): Schwierige Verhältnisse. Interdependenzen zwischen Journalismus und PR, Wiesbaden, 181-195.

Ronneberger, Franz (1977): Legitimation durch Information, Düsseldorf.

Ronneberger, Franz / Rühl, Manfred (1992): Theorie der Public Relations. Ein Entwurf, Opladen.

Röttger, Ulrike (1997): Journalistische Qualifikationen in der Öffentlichkeitsarbeit. Inhaltsanalyse von PR-Stellenanzeigen. In: Bentele, Günter / Haller, Michael (Hg.): Aktuelle Entstehung von Öffentlichkeit. Akteure - Strukturen - Veränderungen. Konstanz, 267-277.

Röttger, Ulrike (2000): Public Relations - Organisation und Profession. Öffentlichkeitsarbeit als Organisationsfunktion. Eine Berufsfeldstudie, Wiesbaden.

Röttger, Ulrike (2002): Kundenzeitschriften: Camouflage, Kuckucksei oder kompetente Information? In: Publizistik 47, Sonderheft 3: Zeitschriften und Zeitschriftenforschung, 109-125.

Sallot, Lynne M. / Steinfatt, Thomas M. / Salwen, Michael B. (1998): Journalists' and Public Relations Practitioners' News Values: Perceptions and Cross-Perceptions. In: Journalism and Mass Communication Quarterly 75, 366-377. 
Schantel, Alexandra (2000): Determination oder Intereffikation? Eine Metaanalyse der Hypothesen zur PR-Journalismus-Beziehung. In: Publizistik 45, 70-88.

Schauer, Reinbert / Purtschert, Robert / Witt, Dieter (Hg.) (2002): Nonprofit-Organisationen und gesellschaftliche Entwicklung: Spannungsfeld zwischen Mission und Ökonomie, Linz.

Scholl, Armin / Weischenberg, Siegfried (1998): Journalismus in der Gesellschaft. Theorie, Methodologie und Empirie, Opladen.

Serini, Shirley A. (1993): Influences on the Power of Public Relations Professionals in Organizations: A Case Study. In: Journal of Public Relations Research 5 (1), 1-25.

Take, Ingo (2002): NGOs im Wandel. Von der Graswurzel auf das diplomatische Parkett, Wiesbaden.

Theis-Berglmair, Anna Maria (2005): Public Relations aus organisationssoziologischer Perspektive. In: Bentele, Günter / Fröhlich, Romy / Szyszka, Peter (Hg.): Handbuch der Public Relations. Wissenschaftliche Grundlagen und berufliches Handeln. Mit Lexikon, Wiesbaden, 37-49.

Weaver, David H. u. a. (2007): The American Journalist in the $21^{\text {st }}$ Century: U.S. News People at the Dawn of a New Millenium, Mahwah, N.J.

Weber, Johanna (1999): Das Verhältnis Journalismus und Öffentlichkeitsarbeit. Eine Forschungsübersicht zu den Eckpunkten einer wiederentdeckten Diskussion. In: Rolke, Lothar / Wolff, Volker (Hg.): Wie die Medien die Wirklichkeit steuern und selber gesteuert werden, Opladen, 265-276.

Weber, Stefan (2004): Gemeinsamkeiten statt Unterschiede zwischen Journalismus und PR. In: Altmeppen, Klaus-Dieter / Bentele, Günter / Röttger, Ulrike (Hg.): Schwierige Verhältnisse. Interdependenzen zwischen Journalismus und PR, Opladen, 53-66.

Weick, Karl E. (1998): Der Prozeß des Organisierens, 2. Aufl., Erstveröffentl. 1969, Frankfurt a. M.

Weischenberg, Siegfried (1995): Journalistik. Theorie und Praxis aktueller Medienkommunikation. Bd. 2: Medientechnik, Medienfunktionen, Medienakteure, Opladen.

Weischenberg, Siegfried / Malik, Maja / Scholl, Armin (2006): Die Souffleure der Mediengesellschaft. Report über die Journalisten in Deutschland, Konstanz.

Willke, Helmut (2000): Systemtheorie I: Grundlagen. Eine Einführung in die Grundprobleme der Theorie sozialer Systeme, 6. Aufl., Stuttgart. 